\title{
Practice Spotlight: Community Intravenous Program, Winnipeg Regional Health Authority
}

\author{
Lesley Hoy, BSc(Pharm) \\ Senior Pharmacist \\ Community Intravenous ProgramWinnipeg \\ Regional Health Authority \\ Winnipeg, Manitoba
}

$\mathrm{T}^{\mathrm{s}}$ 'he Community Intravenous Program (CIVP) is a multidisciplinary program within the Winnipeg Regional Health Authority, with a referral base of over 650000 peoplethe entire city of Winnipeg. The program was started more than 30 years ago at St Boniface General Hospital and evolved into an established home IV therapy program with a cap of 50 clients. To reduce the number of scheduled visits to Winnipeg's 6 emergency departments and its single urgent care centre (for the purpose of IV antibiotic therapy) and to limit delays in hospital discharge (because of the program cap), the Pharmacy, Home Care, and Medicine programs of the Winnipeg Regional Health Authority collaborated in 2006 to extensively expand and relocate the program, now known as the CIVP. This expansion has had a tremendous impact on the role of the CIVP pharmacists, through an increase in pharmacy resources and the development of CIVP-specific pharmacy practice expectations.

Patients who are assessed in an emergency department or the urgent care centre and who require IV administration of antibiotics are referred directly to 1 of the 2 CIVP infusion clinics. At the clinic, the client is assessed by the multidisciplinary team. Patients who have been admitted to hospital but who appear suited to treatment in the community are referred to the CIVP central office for assessment by the multidisciplinary team before discharge. Part of this assessment includes determination of the most appropriate venue for antibiotic administration. Some clients can travel to a clinic, but for others, nursing visits to the home are arranged. At the time of writing, in spring 2009, about 40 clients were being treated at home and about 60 were being treated in the infusion clinics.

The CIVP team consists of a program team manager, a medical director, infectious disease physicians, pharmacists, pharmacy technicians, and nurses. Pharmacy human resources consist of 5 full-time pharmacists and 6 full-time technicians, who are funded through the Winnipeg Regional Health Authority Pharmacy Program.

The CIVP pharmacists provide a range of clinical services and are involved in the education of both clients and members of the multidisciplinary team. The pharmacists identify opportunities for optimization of pharmacotherapy by creating a best possible medication profile for each new client. This profile includes clarification of any allergies, assessment for adverse drug reactions, identification of prior antibiotic use, and review of pertinent laboratory results, including microbial culture. A detailed medical history, including information about the current illness, is obtained and assessed in collaboration with a physician and a nurse. Once the assessment is complete, the pharmacist develops a care plan, which includes monitoring of the safety and efficacy of the antibiotic therapy and consideration of resistance patterns, common pathogens, antibiotic sensitivities, and concomitant disease states. The pharmacists follow laboratory results according to standing orders for blood work and therapeutic drug monitoring within the CIVP. The client's clinical status is regularly reviewed, nephrotoxicity and ototoxicity are assessed as required, and the results of microbial culture are routinely reviewed. Pharmacists also respond to drug information requests from other members of the team and from the 38 CIVP nurses working in the community. In addition, the pharmacists have played an integral role in the development of policies, procedures, and practice guidelines for this expanding program.

Another important role for the CIVP pharmacists is evaluating clients for potential conversion to oral antibiotics. This involves reassessing the client, but before the reassessment, the physician and pharmacist decide upon an appropriate oral agent and follow-up plan, based on the client's anticipated 
situation. Then, under the direction of the infectious disease physician, the pharmacist and nurse reassess some patients for oral step-down therapy. This practice has helped to decrease the number of physician assessments and allows physicians more time for new CIVP admissions.

The role of pharmacists within the Winnipeg Regional Health Authority CIVP continues to evolve. The CIVP pharmacists have a unique practice, as they serve clients from across the entire city of Winnipeg and are allowed to practise independently within a team environment. The relationship among the Infectious Disease, Microbiology, Pharmacy, and Nursing departments allows for shared continuing education, which leads to excellence in the quality of care provided to CIVP clients. The CIVP pharmacists are continuously exploring methods of enhancing their practice. In addition, we are interested in pursing research opportunities and possibly expanding our role beyond the provision of IV antibiotic therapy.

\section{Acknowledgments}

I would like to take this opportunity to acknowledge the support that the CIVP team received from Jamie Falk, BSc(Pharm), PharmD, during the initial expansion of the CIVP pharmacists' role, and to thank Colette Raymond, BSc(Pharm), PharmD, MSC, ACPR, who provided editorial support and encouragement during the writing of this article. Finally, I would like to recognize the CIVP pharmacists and other CIVP team members for their dedicated work during the expansion of the Winnipeg Regional Health Authority CIVP.-Lesley Hoy

The Practice Spotlight series highlights the accomplishments of Canadian pharmacists with unique practices in hospitals and related health care settings. If you have a unique or innovative practice, or you know someone else who should be profiled, please submit your contact information to Mary Ensom, Editor of CJHP (cjhpedit@cshp.ca), and one of our Associate Editors will be in touch with you.

\section{ON THE FRONT COVER}

\section{Role 3 Multinational Medical Unit Kandahar Air Field, Afghanistan}

The Role 3 Multinational Medical Unit, situated at Kandahar Air Field in Afghanistan, is an austere single-story plywood building surrounded by monolithic concrete blast barriers. Do not let the

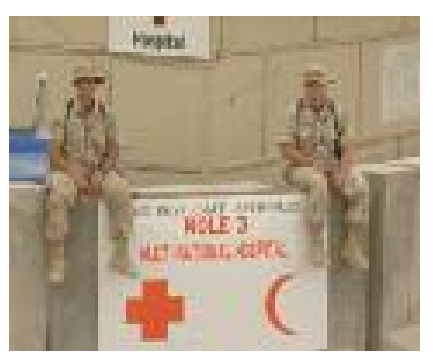
severe exterior fool you, however, for inside you will find a world-class trauma hospital staffed by dedicated professionals of 7 different nationalities: Canadians, Americans, Dutch, Danish, British, New Zealanders, and Australians.

The hospital serves a broad population, from the more than 15000 soldiers and civilian workers on the Kandahar Air Field itself to the tens of thousands of additional coalition soldiers stationed throughout Afghanistan. Care is also provided to Afghans employed in the country's army and police forces and to Afghan civilians who need medical services to save life, limb, or eyesight.
The Role 3 Multinational Medical Unit provides outpatient and inpatient services, with treatment for conditions ranging from the common cold to severe blast injuries caused by improvised explosive devices (also known as IEDs). As of late winter 2009, the hospital boasted 8 trauma bays; a 13-bed ward; a 9-bed intensive care unit; 3 operating rooms; a radiology suite with computed tomography scanner; a medical laboratory; dental, physiotherapy, and primary care clinics; and a pharmacy.

Pictured on the cover are Lieutenant (Navy) Warren Prokopiw, Officer-In-Charge of Clinical Support Services and the Forward Medical Pharmacist, and Captain Allan Edwards, Clinical Pharmacist with the Role 3 Multinational Medical Unit. The picture was taken by Major Tim Barter, Advanced General Dentist, using a Canon EOS 10D camera and Sigma AF lens.

The CJHP would be pleased to consider photographs featuring rural hospitals and their pharmacy departments or residential care facilities taken by CSHP members for use on the front cover of the journal. If you would like to submit a photograph, please send an electronic copy (minimum resolution $300 \mathrm{dpi}$ ) to Sonya Heggart at sheggart@cshp.ca. 